\title{
Corneal Distortion and Corneal Morphological Parameters in Naïve Eyes Analyzed with a Scheimpflug Camera
}

\author{
Sandro Sbrodone ${ }^{1}$, Adele Ragucci ${ }^{1}$, Gennarfrancesco Iaccarino ${ }^{1}$, Domenico de Robertis ${ }^{1}$, Giovan Battista Scazzi ${ }^{1}$ \\ \& Michele Lanza ${ }^{1}$ \\ ${ }^{1}$ Multidisciplinary Department of Medical, Surgical and Dental Specialites, Università della Campania Luigi \\ Vanvitelli, Napoli, Italy \\ Correspondence: Michele Lanza, Multidisciplinary Department of Medical, Surgical and Dental Specialites, \\ Università della Campania Luigi Vanvitelli, Napoli, Italy. Tel: 39-3287-058-652. E-mail: mic.lanza@gmail.com
}

Received: November 24, 2020; Accepted: December 21, 2020; Published: December 30, 2020

The authors report no conflicts of interest. Grant/funding: This project did not receive external funding.

\begin{abstract}
Background: The study of the corneal biomechanical properties (CBP) is gaining always more interest, thanks to the applications to refractive surgery and to the overall management of several corneal diseases.

Purpose: To analyze the eventual relationships between corneal distortion and morphologic features in healthy subjects.

Methods: one eye of one hundred and fifty-two healthy participants, 87 women and 65 men, aging from 23 to 65 years old, were evaluated in this study. Every eye underwent a complete clinical examination, that included also a tomographic scan using Pentacam HR (Oculus, Wetzlar, Germany) and a biomechanical evaluation using Corvis ST (Oculus, Wetzlar, Germany). Linear regressions among refractive defect, corneal volume (CV), anterior corneal curvature and central corneal thickness (CCT) vs corneal biomechanical features provided by Corvis ST have been ran using SPSS software version 18.0.

Results: Corneal distortion factors showed a low correlation both with corneal morphological parameters and with refractive defect. No significant correlations ( $p>0.01$ ) were found among morphologic and biomechanical features evaluated.

Discussion: results observed in this study could help in recognize the healthy range of values of CBP parameters provided by CST. This information could be very useful in many ophthalmology topics such as the accurate IOP measurement, the early diagnosis of corneal degenerations, such as keratoconous, and the screening of subject undergoing refractive surgery.
\end{abstract}

Conclusions: even if data of this study need to be confirmed in further ones with larger populations, results here observed suggest that Corvis ST is a device providing an evaluation of corneal distortion not influenced by morphological corneal factors. This information could help in better screening and managing the diseased corneas.

Keywords: corneal biomechanical evaluation, naive eyes, corneal distortion, tomography, Scheimpflug camera

\section{Introduction}

Evaluation of corneal biomechanical properties (CBP) is increasing its importance in many aspects of ophthalmology, mainly in corneal diseases and transplants [1,2], in overall eyeball deformation analysis [3], in refractive surgery [4] and in intraocular pressure evaluation [5]. Devices currently able to provide CBP evaluation are the Ocular Response Analyzer (ORA) (Reichert Ophthalmic Instruments, NY, USA) [6] and the Corvis ST (CST) (Oculus, Wetzlar, Germany) [7,8]. To improve the knowledge of the details of both corneal and eyeball deformation could lead in a better understanding the details of corneal behaviors in cases of external stimuli, such as the surgical ones, and of the internal ones [5]. These are the reasons because CBP are largely investigated in many kinds of eyes, aiming to provide better indications for diagnosis and management of corneal diseases or better screening for corneal surgery [5-9].

In order to better standardize the information coming from new instruments, it is important to evaluate them on naïve eyes. This could help to identify the borders between diseased and not diseased values of CBP parameters. 
The first studies published about CST regarded reliability, reproducibility, and correlations with other ocular parameters in naïve eyes $[10,11]$, but they didn't provide unanimous results.

Purpose of this study is to contribute to better understanding of corneal deformation process analyzed by CST evaluating the eventual relationships between corneal distortion and morphologic features in healthy subjects.

\section{Method}

In this retrospective study, was included only one eye of 152 subjects ( 87 women and 65 men) with a mean age of $35.12 \pm 9.58$ years (range from 23 to 65 years old) and a mean refraction of $-0.45 \pm 1.71 \mathrm{D}$, with a range going from $-7 \mathrm{D}$ to $+3 \mathrm{D}$, measured as spherical equivalent. These subjects referred to our Unit to undergo refractive surgery from January 2019 to December 2019. Criteria of exclusion for this study were all those systemic diseases that could interfere with corneal evaluation and the presence of ocular comorbidities. Participants were asked to stop using contact lens at least one week before every visit. Each of them underwent a complete eye visits and both a Pentacam HD and Corvis ST scan (Oculus, Wetzlar, Germany) to assess the corneal morphological details and the CBP. Pentacam HD is a Scheimpflug camera based, corneal tomographer always more used in the clinical practice to screen and manage corneal diseases $[12,13]$. The device is able to provide several corneal features, the ones evaluated in this study were the following: anterior corneal curvature measured with Sim'K (SK), corneal volume (CV) and central corneal thickness (CCT) at pupil center. Corvis ST is able to record the corneal behavior during an air puff indentation thanks to a Scheimpflug camera able to process 4330 images per second, and it shows many corneal deformation parameters that are well defined (Figure 1) [5]; this study evaluated all of them. Three good quality CST measurements have been performed and every scan has been performed after 5 minutes of rest; the Pentacam scan was administrated always before CST exam and Goldmann applanation tonometry. Normality of distribution was tested with Kolmogorov-Smirnov test, linear Pearson correlation index was selected after Kolmogorov-Smirnov test was applied to the sample and a normal distribution was verified. In this way were evaluated the correlations among morphological parameters such as SK, CCT and CV, the refraction (SE), and the parameters provided by Corvis ST about the corneal distortion. A SPSS software version 18.0 (IBM Corp. Armonk, New York) has been used for statistical evaluation. In this study, only the right eyes of participants were included in the statistical elaboration, whereas the evaluation included always both ones, aiming to avoid bias related to the inner correlations between paired organs that could influence the statistical results.

This study has been approved by Ethics Committee of Università della Campania Luigi Vanvitelli as retrospective study and it followed the principles of the Declaration of Helsinki. An informed consent regarding the own data use was obtained by every participant.

\section{Results}

Parameters evaluated in this study are summarized in Table 1, they are: mean, range and standard deviation of age, spherical equivalent refraction, CCT, CV, SK, and CST parameters: first applanation time (AT1); first applanation length (AL1); first applanation velocity (AV1) second applanation time (AT2); second applanation length (AL2); second applanation velocity (AV2), highest concavity time (HCT); highest concavity peak distance (HCPD); highest concavity peak radius (HCPR); highest concavity deformation amplitude (HCDA).

None of the relationship evaluated showed significant correlation, with $p$ value $>0.01$ in every cases (Table 2,3). Comparing the different CCT values provided by CST (mean $563.45 \pm 27.45 \mu \mathrm{m}$ ) and Pentacam (mean: $558.2 \pm$ $34.24 \mu \mathrm{m})$, no statistical difference has been found $(\mathrm{p}=0.34)$.

In order to deeper analyze the relationships between corneal deformations and corneal morphological parameters, the following correlations have been investigated: difference between AT1 and AT2 (ATD); ratio between AT1 and CCT provided by Pentacam (ATCCTR); ratio between AT1 and HCT (ATHCTR); HCR (ATHCRR) with SE and corneal morphological parameters. Results are summarized in table 3.

The lack of significant correlations between the evaluated parameters suggest that CBP features provided by CST could be independent by the corneal morphological details studied and by the refraction.

Table 1. Mean, standard deviation (SD) and range of the parameters evaluated in this study. SE: Spherical equivalent; SK: anterior corneal curvature measured with Sim'K; CCT: central corneal thickness.

\begin{tabular}{lll}
\hline Parameters & Mean \pm SD & Range \\
\hline Age (years) & $35.12 \pm 9.58$ & $22-67$ \\
SE (D) & $-0.45 \pm 1.71$ & $-8.13-+3.75$ \\
SK (D) & $42.87 \pm 1.36$ & $40.5-45.90$ \\
\hline
\end{tabular}




\begin{tabular}{lll}
\hline Corneal volume $(\mathrm{mm} 3)$ & $61.71 \pm 3.14$ & $54.3-69.70$ \\
CCT $(\mu \mathrm{m})$ & $558.20 \pm 34.24$ & $484-642$ \\
Applanation time 1 $(\mathrm{ms})$ & $7.27 \pm 0.33$ & $6.83-9.15$ \\
Applanation length 1 $(\mathrm{mm})$ & $1.69 \pm 0.28$ & $1.27-2.31$ \\
Applanation velocity 1 $(\mathrm{m} / \mathrm{s})$ & $0.16 \pm 0.04$ & $0.04-0.24$ \\
Applanation time 2 $(\mathrm{ms})$ & $21.43 \pm 0.39$ & $20.21-22.23$ \\
Applanation length 2 $(\mathrm{mm})$ & $1.93 \pm 0.49$ & $0.92-2.74$ \\
Applanation velocity 2 $(\mathrm{m} / \mathrm{s})$ & $-0.32 \pm 0.13$ & $-0.30--0.52$ \\
Deformation amplitude to the highest concavity $(\mathrm{mm})$ & $1.04 \pm 0.12$ & $0.70-1.31$ \\
Highest Concavity Time $(\mathrm{ms})$ & $16.51 \pm 0.57$ & $13.5-17.56$ \\
Highest Concavity Peak Distance $(\mathrm{mm})$ & $4.68 \pm 0.31$ & $3.88-5.32$ \\
Highest Concavity Peak Radius $(\mathrm{mm})$ & $7.02 \pm 0.91$ & $0.72-1.29$ \\
\hline
\end{tabular}

Table 2. Pearson Correlation Index $\left(\mathrm{R}^{2}\right)$ between Corneal Deformation Parameters versus Corneal Morphological Parameters and Spherical Equivalent (SE). CCT: central corneal thickness; CV: Corneal volume; SK: anterior corneal power measured with Sim'K.

\begin{tabular}{lllll}
\hline & $\mathrm{SE}$ & $\mathrm{SK}$ & $\mathrm{CCT}$ & $\mathrm{CV}$ \\
& $\mathrm{R}^{2}$ & $\mathrm{R}^{2}$ & $\mathrm{R}^{2}$ & $\mathrm{R}^{2}$ \\
\hline Applanation time1(ms) & 0.0215 & 0.1259 & 0.312 & 0.0955 \\
Applanation length1(mm) & 0.0008 & 0.0054 & 0.0006 & 0.0224 \\
Applanation velocity1(m/s) & 0.0394 & 0.216 & 0.029 & 0.0011 \\
Applanation time2(ms) & 0.0547 & 0.3006 & 0.092 & 0.00261 \\
Applanation length2(mm) & 0.0598 & 0.1397 & 0.051 & 0.035 \\
Applanation Velocity2(m/s) & 0.0426 & 0.2981 & 0.122 & 0.0061 \\
Deformation amplitude at the highest concavity $(\mathrm{mm})$ & 0.1987 & 0.3241 & 0.209 & 0.0367 \\
Highest Concavity Time $(\mathrm{ms})$ & 0.0056 & 0.3961 & 0.0072 & 0.1065 \\
Highest Concavity Peak Distance $(\mathrm{mm})$ & 0.0021 & 0.1764 & 0.0503 & 0.5521 \\
Highest Concavity Peak Radius $(\mathrm{mm})$ & 0.0452 & 0.0342 & 0.0229 & 0.3418 \\
\hline
\end{tabular}

Table 3. Pearson Correlation Index $\left(\mathrm{R}^{2}\right)$ between difference between AT1 and AT2 (ATD); ratio between AT1 and CCT provided by Pentacam (ATCCTR); ratio between AT1 and HCT (ATHCTR); HCR (ATHCRR) versus Corneal Morphological Parameters and Spherical Equivalent (SE). CCT: central corneal thickness; CV: Corneal volume; SK: anterior corneal power measured with Sim'K.

\begin{tabular}{lllll}
\hline & $\mathrm{SE}$ & $\mathrm{SK}$ & $\mathrm{CCT}$ & $\mathrm{CV}$ \\
& $\mathrm{R}^{2}$ & $\mathrm{R}^{2}$ & $\mathrm{R}^{2}$ & $\mathrm{R}^{2}$ \\
\hline ATD $(\mathrm{ms})$ & 0.0697 & 0.0358 & 0.0213 & 0.1038 \\
ATCCR & 0.0442 & 0.0334 & 0.0543 & 0.0973 \\
ATHCTR & 0.0217 & 0.0101 & 0.0058 & 0.0671 \\
ATHCRR & 0.0342 & 0.0697 & 0.0417 & 0.0284 \\
\hline
\end{tabular}

\section{Discussion}

CBP analysis is gaining always more interest in order to better understand the corneal behavior both after external stimuli, such as surgical ones, and during some corneal diseases that could induce ultra-structural modifications [1-5]. Corvis ST is the first device able to provide both real time imagines and parameters related to corneal deformation [10,11]. Papers about CBP evaluation in different kind of eyes have been previously published [1-5] without reaching unanimous conclusions. Moreover, the authors of this study believe that it is important to start the validation of a new device estimating healthy eyes. It is important to highlight that Corvis St is the second device able to perform this kind of analysis in human eyes and it works in a completely different way compared to the other instrument able to perform corneal biomechanics analysis: ORA [6,10].

No statistical correlations were observed in this study among the refraction, the morphological features of the corneal usually evaluated such as CCT, CV, SK and the ones related to CBP provided by Corvis ST measured in healthy subjects. These results could be explained by two main theories: a) the device is not providing reliable and 
accurate measurements of corneal distortion or b) it could mean that morphological and refractive parameters evaluated are not affecting the CST measurement of CBP.

Data observed in this study could be used to identify the range of healthy eye's parameters aiming to increase the usefulness of corneal distortion evaluation.

Results observed in this study were different from the ones reported by Hon [10] regarding correlation analysis because they found HCDA negatively correlated with CCT. The reasons of this different could be more than one: 1) this study has been conducted evaluating a larger sample; 2) subject enrolled in this study have been asked to stop using contact lens at least one week before the eye-visit whereas Hon et al. [10] included participants not wearing them only in the previous 24 hours; 3 ) in this study the tomography with Pentacam was always administrated before corneal distortion measurement performed with CST, aiming to avoid introducing biases related to measurements of parameters such as SK whereas, Hon et al. [10] adopted a random order for morphological and CBP evaluation. In this way, the air puff produced by CST could determine a misevaluation of morphological corneal parameters. First results about reliability of IOP evaluation with CST observed by Hon [10] are different by the Hong [11] ones; it could be due to the different study population they enrolled or because some of the diverse details in methodology of the studies. Even if the first papers published about CST evaluation in healthy eyes are from some years ago [10-13], the interest about the evaluation of this device in this kind of eye is still present [14-18]. Miki et al. published some interesting papers about this topic, in one of them evaluating only healthy eyes [14], they analyzed 96 healthy subjects observing strong correlations between CBP parameters with axial length, IOP and age. Some very softer relationships were noted with CCT and corneal curvature [14]. In the ones published by same group more recently [15], they compared healthy eyes with glaucomatous one evaluating the CBP provided by CST, they found a significant difference between two groups but correlations with IOP, CCT, age and axial length have been studied only in glaucoma eyes. Fuchsluger et al. [15] compared CST parameters in patients affected by keratoconous, patient undergone corneal crosslinking for keratoconous and healthy subjects. In their study, in healthy subjects, they found a correlation between AV1, AT1, AV2 and HCDA with CCT. The different results observed in this study could be related to some details related to the populations study: a) the number of eyes included (50 vs 152); b) the age range (21-41 vs 22-67) [15]. Moreover, different version of Pentacam and CST have been used. Herber et al. [16] evaluated CST and ORA in both healthy subjects and keratoconous eyes, in first group they found strong correlations between many CST parameters and thinnest corneal thickness and a weaker one with maximum keratometry but in this study different features have been analyzed. In their interesting paper, Zhang et al. [18] evaluated new parameters provided by new version of CST in evaluating specifically the corneal stiffness in myopic eyes and observed a correlation among them and morphological ones such as CCT and IOP. Differences with our study are mainly related to the selection of the subjects included: in this one, no restrictions related to refractive defects were applied. The understanding of the overall corneal deformation process in healthy eyes still has some aspect to clarify and this study provide a contribute to this topic. To completely understand the corneal behaviors to different kind of stimuli could be extremely important to develop models for more accurately measure IOP, also in eyes with nor regular corneas, to earlier detect corneal disease, such as keratoconous, that could benefit by earlier treatment, and to better screen patients for refractive surgery. This is the reason because, in this study. additional parameters, obtained by the difference or the ratio of some of the features provided by CST have been evaluated, still observing no significant correlations with CBP. Relationship between CBP provided by CST and IOP have been studied and clear information about this are not still reached, the reason of this could be due to the novelty of the CST, that is a new and evolving device, and it needs to be evaluated in different kind of patients. Some features, such as AT1, seems strongly correlated with IOP but further and specific studies are needed to have more accurate information [1920].

\section{Conclusions}

Even if parameters currently provided by CST are just applied to clinical practice, to improve the quality of management of patients [21], it is important to continue to evaluate the CBP and the corneal deformation process in order to better standardize information coming by this new and very interesting device. Thus, information coming from this study need to be confirmed in further ones with larger populations, to have more accurate details regarding corneal deformation, independent from morphological corneal characteristics. Values provided by this study, together with the other ones previously published, would be useful also to recognize healthy and suspect values of CBP. 


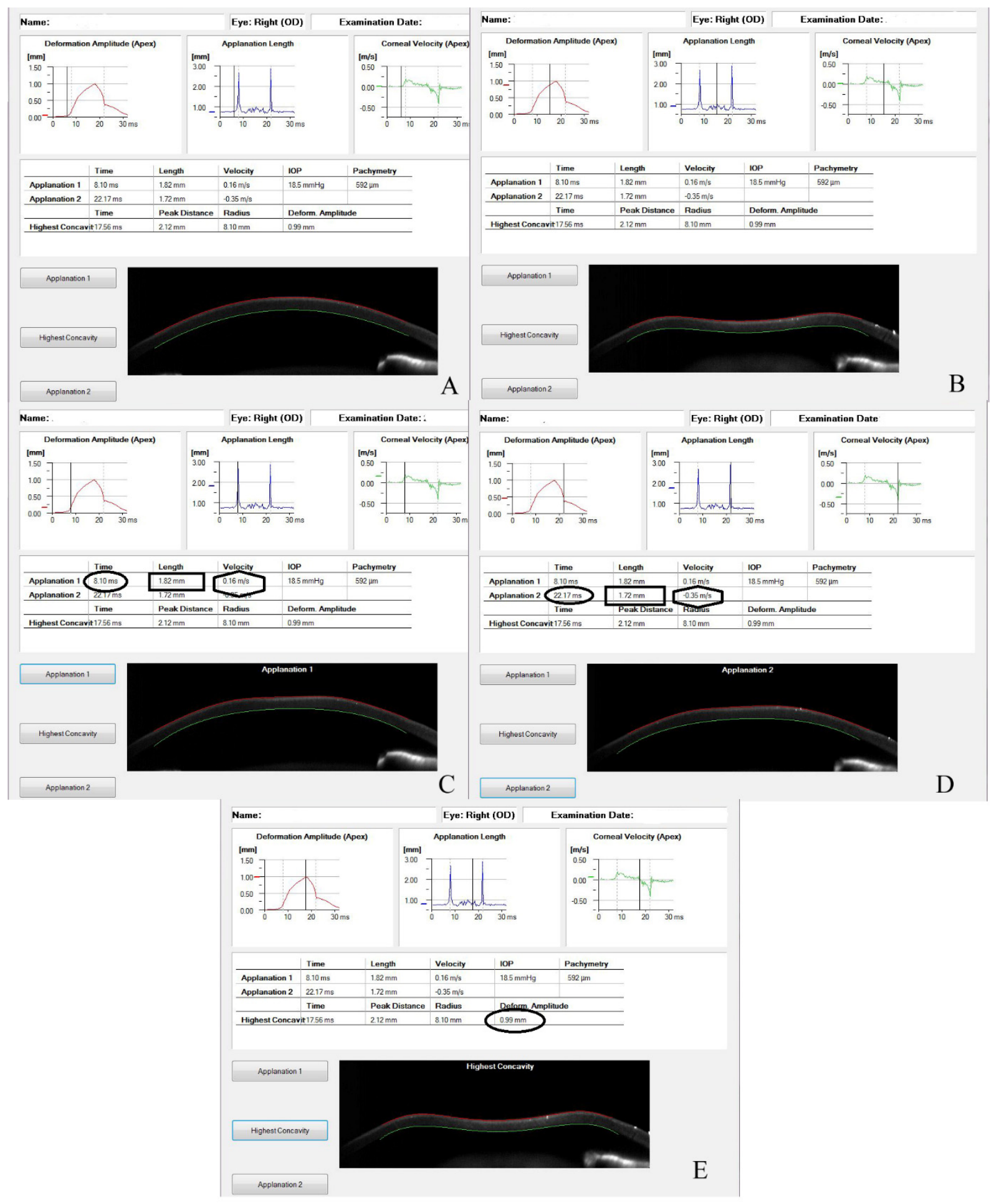

Figure 1. Screenshot of Corvis ST display, showing information recorded immediately upon the air impulse (A); screenshot of Corvis ST display, showing information recorded during the corneal deformation obtained by the air impulse (B); screenshot of Corvis ST display, showing Time of Applanation 1 (ellipse), Length of Applanation 1 (rectangle), Velocity of Applanation 1 (hexagon) at first applanation (C); screenshot of Corvis ST display, showing Time of Applanation 2 (ellipse), Length of Applanation 2 (rectangle), Velocity of Applanation 2 (hexagon) at second applanation (D); screenshot of Corvis ST display, showing Deformation Amplitude at the highest concavity at corneal apex (ellipse) (E) 


\section{References}

[1] Abdelkader, A. (2013). Influence of different keratoplasty techniques on the biomechanical properties of the cornea. Acta Ophthalmol, 91, e567-272. https://doi.org/10.1111/aos.12136

[2] Abd, E. M. S., Elsobky, H. M., Zaky, A. G., Hassan, E. A. M., \& Khalaf, Allah, M. T. (2019). Corneal biomechanics and intraocular pressure assessment after penetrating keratoplasty for non keratoconic patients, long term results. BMC Ophthalmol, 19, 172. https://doi.org/10.1186/s12886-019-1186-y

[3] Koprowski, R., \& Wilczyński, S. (2018). Corneal vibrations during intraocular pressure measurement with an air-puff method. J. Healthc. Eng, 11, 5705749. https://doi.org/10.1155/2018/5705749

[4] Guo, H., Hosseini-Moghaddam, S. M., \& Hodge, W. (2019). Corneal biomechanical properties after SMILE versus FLEX, LASIK, LASEK, or PRK: a systematic review and meta-analysis. BMC Ophthalmol, 19, 167. https://doi.org/10.1186/s12886-019-1165-3

[5] Lanza, M., Iaccarino, S., \& Bifani, M. (2016). In vivo human corneal deformation analysis with a Scheimpflug camera, a critical review. J. Biophotonics, 9, 464-477. https://doi.org/10.1002/jbio.201500233

[6] Luce, D. A. (2005). Determining in vivo biomechanical properties of the cornea with an ocular response analyser. J. Cataract. Refract. Surg, 31, 156-162. https://doi.org/10.1016/j.jcrs.2004.10.044

[7] Hong, J., Xu, J., Wei, A., Deng, S. X., Cui, X., Yu, X. et al. (2013). A new tonometer--the Corvis ST tonometer: clinical comparison with noncontact and Goldmann applanation tonometers. Invest. Ophthalmol. Vis. Sci, 54, 659-665. https://doi.org/10.1167/iovs.12-10984

[8] Lanza, M., Cennamo, M., Iaccarino, S., Romano, V., Bifani, M., Irregolare, C. et al. (2015). Evaluation of corneal deformation analysed with a Scheimpflug based device. Cont. Lens. Anterior Eye, 38, 89-93 https://doi.org/10.1016/j.clae.2014.10.002

[9] Ma, J., Wang, Y., Wei, P., \& Jhanji, V. (2018). Biomechanics and structure of the cornea: implications and association with corneal disorders. Surv. Ophthalmol, 63, 851-861. https://doi.org/10.1016/j.survophthal.2018.05.004

[10] Hong, J., Xu, J., Wei, A., Deng, S. X., Cui, X., Yu, X. et al. (2013). A New Tonometer-The Corvis ST Tonometer: Clinical Comparison with Noncontact and Goldmann Applanation Tonometers. Invest. Ophthalmol. Vis. Sci, 54, 659-665. https://doi.org/10.1167/iovs.12-10984

[11] Hon, Y., \& Lam, A. K. (2013). Corneal deformation measurement using scheimpflug noncontact tonometry. Optom. Vis. Sci, 90, e1-8. https://doi.org/10.1097/OPX.0b013e318279eb87

[12] Huseynova, T., Abdulaliyeva, F., \& Lanza, M. (2016). Comparison of Scheimpflug imaging parameters between steep and keratoconic corneas of Caucasian eyes. Clin. Ophthalmol, 10, 603-608. https://doi.org/10.2147/OPTH.S102683

[13] Lanza, M., Iaccarino, S., Mele, L., Carnevale, U. A., Irregolare, C., Lanza, A. et al. (2016). Intraocular pressure evaluation in healthy eyes and diseased ones using contact and non contact devices. Cont. Lens. Anterior Eye, 39, 154-159. https://doi.org/10.1016/j.clae.2015.10.001

[14] Miki, A., Maeda, N., Ikuno, Y., Asai, T., Hara, C., \& Nishida, K. (2017). Factors associated with corneal deformation responses measured with a dynamic Scheimpflug analyzer. Invest. Ophthalmol. Vis. Sci, 58, 538-544. https://doi.org/10.1167/iovs.16-21045

[15] Miki, A., Yasukura, Y., Weinreb, R. N., Yamada, T., Koh, S., Asai, T. et al. (2019). Dynamic Scheimpflug ocular biomechanical parameters in healthy and medically controlled glaucoma eyes. J. Glaucoma, 28, 588592. https://doi.org/10.1097/IJG.0000000000001268

[16] Fuchsluger, T. A., Brettl, S., Geerling, G., Kaisers, W., \& Franko, Zeitz, P. (2019). Biomechanical assessment of healthy and keratoconic corneas (with/without crosslinking) using dynamic ultrahigh-speed Scheimpflug technology and the relevance of the parameter (A1L-A2L). Br. J. Ophthalmol. 103, 558-564.

https://doi.org/10.1136/bjophthalmol-2017-311627

[17] Herber, R., Ramm, L., Spoerl, E., Raiskup, F., Pillunat, L. E., \& Terai, N. (2019). Assessment of corneal biomechanical parameters in healthy and keratoconic eyes using dynamic bidirectional applanation device and dynamic Scheimpflug analyzer. J. Cataract Refract. Surg, 45, 778-788. https://doi.org/10.1016/j.jcrs.2018.12.015

[18] Zhang, Y., Wang, Y., Li, L., Dou, R., Wu, W., Wu, D. et al. (2018). Corneal stiffness and its relationship with 
other corneal biomechanical and nonbiomechanical parameters in myopic eyes of Chinese patients. Cornea, 37, 881-885. https://doi.org/10.1097/ICO.0000000000001605

[19] Salouti, R., Bagheri, M., Shamsi, A., \& Zamani M. (2020). Corneal parameters in healthy subjects assessed by Corvis ST. J. Ophthalmic Vis. Res,. 15, 24 - 31. https://doi.org/10.18502/jovr.v15i1.5936

[20] Garcia-Feijoo, J., Martinez-de-la-Casa, J. M., Morales-Fernandez, L., Saenz-Frances, F., Santos-Bueso, E., Sofia, Garcia-Saenz, S. et al. (2015). New technologies for measuring intraocular pressure. Prog Brain Res, 221, 67 - 79. https://doi.org/10.1016/bs.pbr.2015.06.003

[21] Jędzierowska, M., \& Koprowski, R. (2019). Novel dynamic corneal response parameters in a practice use: a critical review. Biomed. Eng. Online, 18, 17. https://doi.org/10.1186/s12938-019-0636-3

\section{Copyrights}

Copyright for this article is retained by the author(s), with first publication rights granted to the journal.

This is an open-access article distributed under the terms and conditions of the Creative Commons Attribution license (http://creativecommons.org/licenses/by/4.0/). 